\title{
VSearch Color: Full-color visual search experiments on the Macintosh II
}

\author{
JAMES T. ENNS and RONALD A. RENSINK \\ University of British Columbia, Vancouver, British Columbia, Canada
}

\begin{abstract}
We describe an update to our visual search software for the Macintosh line of computers. The new software, VSearch Color, gives users access to the full-color capabilites of the Macintosh II line. One of the key features of the new software is its ability to treat graphics information separately from color information. This makes it easy to study color independently of form, to design experiments based on isoluminant stimuli, and to incorporate texture segregation, visual identification, number discrimination, adaptation, masking, and spatial cuing into the basic visual search paradigm.
\end{abstract}

Last year we introduced a software package that allowed researchers and teachers to use the Macintosh line of computers for experiments in visual search. At that time we described our goals in designing the software, illustrated a wide range of visual search experiments that could easily be implemented with the software (Enns, Ochs, \& Rensink, 1990), and described how to accomplish millisecond timing and display on the Macintosh (Rensink, 1990).

This year we introduce an updated version of the software that (1) permits use of the full-color capability of the Macintosh II line of computers, and (2) enables several new variants of visual search tasks to be run, including texture segmentation, visual identification, number discrimination, masking, and spatial cuing. In this paper, we will highlight the central features of VSearch Color, discuss the steps involved in running an experiment with it, and demonstrate its range by summarizing several types of experiments that can easily be carried out with it.

\section{Features of VSearch Color}

VSearch Color is an extension of VSearch (Enns et al., 1990) that is completely consistent with the spirit and design of the original program. The Imaker program now allows any color image that can be drawn or imported to one of the popular Macintosh color graphics applications (e.g., PixelPaint, PhotonPaint, SuperPaint) to be used as a stimulus. These images may contain up to 256 different colors or levels of gray. Images are copied from the graphics application to the Macintosh clipboard. From there, Imaker is used to create a VSearch-readable file of the image. Since both Imaker and VSearch Color can now be used while MultiFinder is running, all programs can be run concurrently, allowing experimental stimuli to be quickly and easily created and tested.

Correspondence may be addressed to J. T. Enns, Department of Psychology, University of British Columbia, Vancouver, British Columbia V6T 1Y7, Canada.

Note: One or more of the authors has a direct financial interest in some of the software or hardware described in this paper.-Editor
One of the most important additions to VSearch Color is the Palette dialogue box, shown in Figure 1. This window gives the user control over the color environment (i.e., the colors available for the pixels in the displays). Because the Macintosh II separates the color environment from the graphics information contained in an image (i.e., the spatial arrangement of pixels), the user may combine the form and color of an item in a large number of ways. Changing the color environment allows the particular colors associated with an item to be altered even after it has been imported to VSearch Color, something we have found quite useful for purposes such as the design of experiments involving isoluminant stimuli.

VSearch Color also has a new dialogue box that facilitates the testing of apparent isoluminance. In the Isoluminance box, shown in Figure 2, the user has complete access to the Macintosh color system. After two colors have been chosen from the palette, isoluminance can be tested in one of two ways: with the minimal border method, or with the minimal flicker method. The first method simply involves placing the two colors side by side in a box and then adjusting the luminance of one of the colors with the mouse until the border between them is as weak as possible. With the second method, the colors are flashed synchronously over the entire screen or over one half of the screen. The luminance of one of the colors is then varied until the apparent flicker on the screen is reduced as much as possible. Users have control over the frequency of each test flash (maximum frequency of the test flashes is $33.5 \mathrm{~Hz}$ on a standard monitor) and over the duration of the flicker episode. This frequency is well above the $20-\mathrm{Hz}$ minimum generally used for isoluminance testing.

Another color-related addition is the expansion of available background colors and patterns in the Backgrounds dialogue box, shown in Figure 3. Previously, VSearch users were able to choose one of the 38 Macintosh desktop patterns as the background against which search items were placed. These backgrounds included a simulation of brightness levels ranging from white (all pixels lit) to gray ( $50 \%$ 


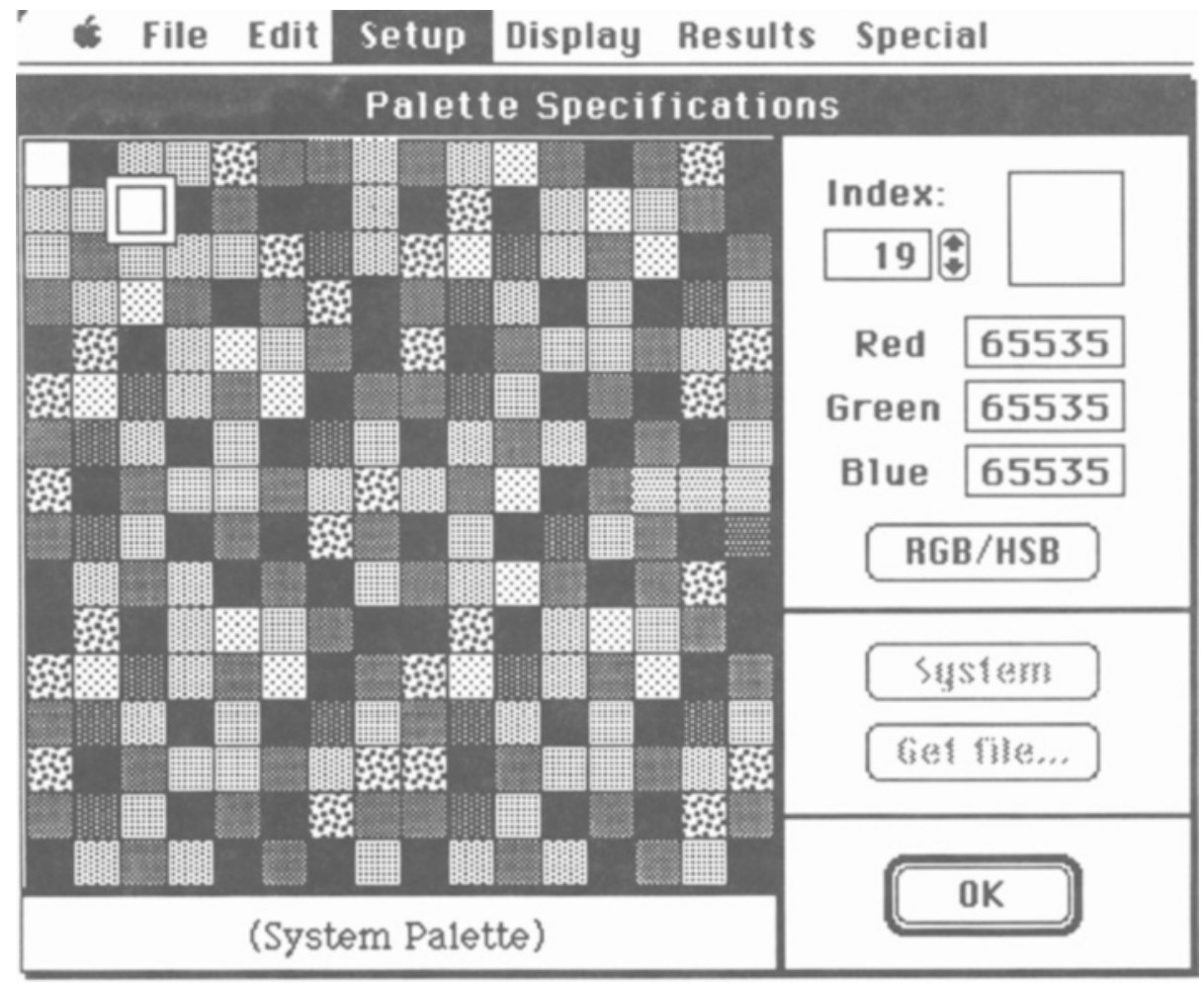

Figure 1. The Palette dialogue box. The color environment of the Macintosh is controlled via this window.

of pixels lit) to black (no pixels lit). VSearch Color still permits choice among the 38 desktop patterns, but, in addition, it is now possible to associate each of the two values of the pattern (forecolor and backcolor) with any of the millions of colors available on the Macintosh II.

Finally, VSearch Color now permits the testing of a much larger range of variants on the basic visual search paradigm. These options can be chosen in the General

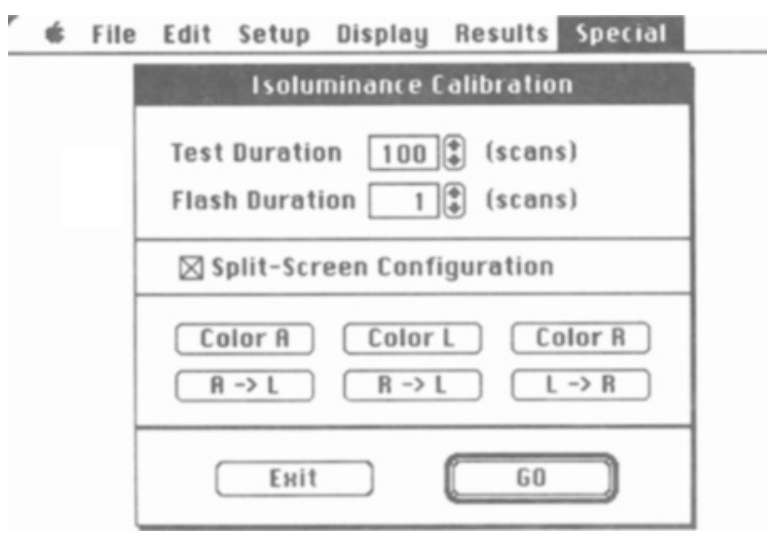

Figure 2. The Isoluminance dialogue box. Two methods of testing for isoluminance are supported: the minimal border method and the minimal flicker method. Users have access to all of the millions of colors available on the Macintosh through the standard Macintosh color-picker. dialogue box, shown in Figure 4. In all, three different types of search tasks (presence/absence, item identification, region identification) can be combined with two different types of search objects (single item, region of items), and six different types of relations between target and distractor items (specific targets and distractors are linked/not linked, the target items present on a given trial are $\mathrm{mixed} / \mathrm{not}$ mixed, the distractor items on a given trial are mixed/not mixed). This allows for a very large sample space of possible experiments. For example, a numberdiscrimination paradigm can be set up by choosing the region-identification task and then assigning a two-item region to one response and a three-item region to the other response. A visual identification paradigm can be set up by choosing the item-identification task.

\section{How to Design and Run an Experiment}

Stimulus items. Any Macintosh graphics file can be used as the source for a stimulus item. This includes color or black and white photographs that have been digitized, images digitized from frames of videotape, images generated by 3-D CAD programs, and images drawn with one of the many color paint programs on the market. Most color graphics editors permit images to be imported and exported through the clipboard. Imaker takes these images and converts them into files that can be used by VSearch Color in several ways. It can use the color palette information without reading the graphics information, it can use both the color and graphics information, or it can 


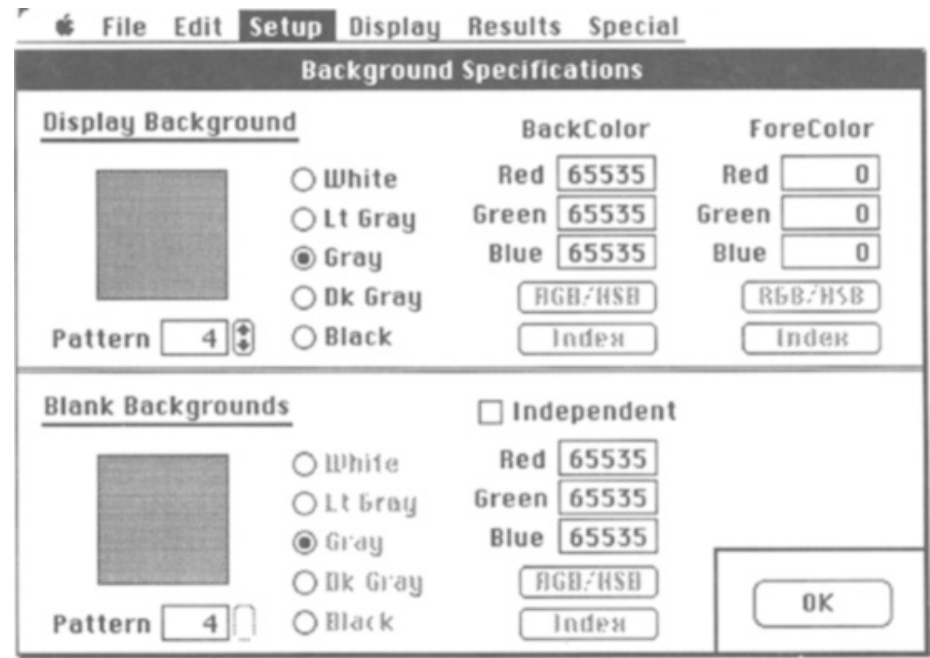

Figure 3. The Backgrounds dialogue box. Each of the 38 Macintosh desktop patterns can be used as the background against which search items are placed. The forecolor and backcolor may each be associated with any of the Macintosh colors.

use the graphics information together with color information from another file.

Experimental design. Decisions about experimental design are made through nine mouse-driven dialogue boxes. The first of these, General, controls the distribution of target and distractor items within a display, the type of search object, the number of experimental trials and blocks of trials, and whether the trial sequence is fixed or variable within each block.

The Sizes dialogue box sets the number of screen rows and columns in which items can appear, the amount of random jitter of each item at a given location, and the

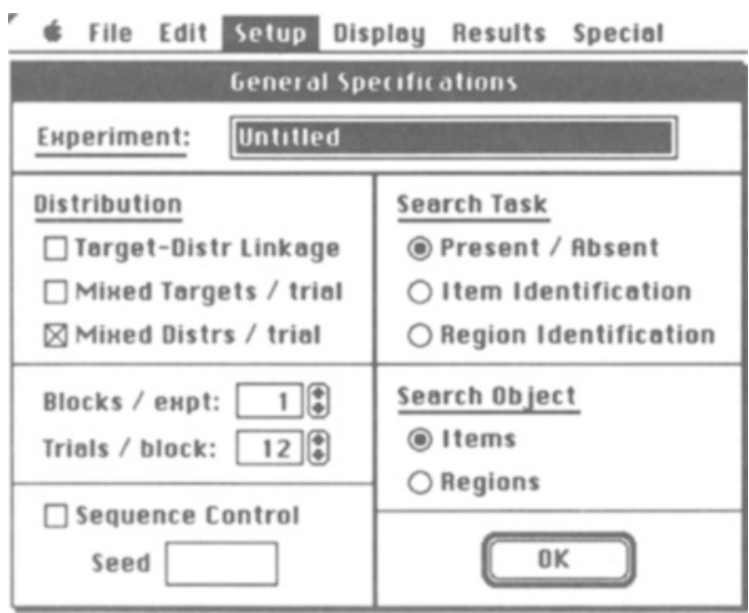

Figure 4. The General dialogue box. Three different types of search tasks can be combined with two different types of search objects and six different types of target-distractor relations, for a very large sample space of possible experiments. number of display items that can appear in a given trial. Up to three subarrays can also be used to control the density of items in a display.

The Duration dialogue box sets the temporal characteristics of the trial. Each trial is divided into nine temporal divisions or "phases," each of which can be set independently. This allows temporal control over such things as the predisplay, display, and postdisplay events (see, e.g., the speed-accuracy tradeoff experiment in the next section). One very useful feature of this dialogue box is the specification of up to eight interval durations for any given trial phase. These intervals are then presented randomly within a trial block.

The Palette dialogue box permits the creation and use of a color palette containing up to 256 different colors. These colors can be chosen from among the millions of colors supported by the Macintosh II. When a new color palette is created, VSearch attempts to map the existing colors to their "nearest" values in the new color palette. This gives the program great flexibility. For example, in an experiment that depends on isoluminant colors, each subject can be tested on the identical graphics items, with the color palette tailored to the color vision characteristics of each subject.

The Item, Mask|Cue, and Backgrounds dialogue boxes set the search items, pre- and postdisplay masks (if any), and background against which items are presented. In each of these dialogue boxes, the graphics and color information may be specified independently. The Mask|Cue dialogue box also allows users to specify whether the chosen item is to be used as a mask or as a location cue.

The Tones dialogue box permits a tone to be placed in the temporal position of the pre- and/or postdisplay mask. The pitch and duration of the tone can be varied directly 
by the user in this box, whereas the volume is controlled via the system Control Panel.

The Fixation dialogue box sets a fixation point and/or a feedback symbol to appear in the intertrial interval. Users control the presence, color (three levels of gray), and size of these symbols in this box, whereas the temporal durations of these events are controlled in the Durations box.

Statistical analysis. VSearch Color stores data in a very compact form. Each response is recorded with 2 bytes, so that thousands of data points can be stored using only a few kilobytes of memory. Data from saved experiments are accessible in one of two forms: raw statistics and summary statistics. Raw statistics (i.e., a trial-by-trial account of display characteristics along with response time and accuracy) can be saved as text files or output on a printer. If placed into text files, the data can be read directly by any of the statistical packages available for the Macintosh (e.g., SuperAnova, StatView, CLR Anova). Summary statistics (i.e., mean response time and accuracy, slopes of search time as a function of display size) may be displayed on screen, printed, and also saved as text files.

\section{VSearch Color Demonstration Displays}

In this section, we provide several examples of displays taken from experiments run with VSearch Color. These examples are by no means exhaustive; they are meant only to illustrate the way in which the program can take advantage of the many graphics applications available for Macintosh computers. (Note that since color images can- not be printed in this journal, different gray levels will be used in all the examples in place of real colors.)

Search for color and conjunctions of color and form. Target items that differ in color from distractor items can be found rapidly in a display, independently of the number of distractor items present (Treisman \& Gelade, 1980). This is the classic demonstration of "pop-out" in visual search, one of the standard diagnostic tests for the primitive features of vision. In contrast, target items defined by a conjunction of color and form (e.g., red X) take longer to find among distractors that share only one of these features (e.g., red $\mathrm{O}$, green $\mathrm{X}$ ). Here there is a linear relation between response time and display size. Furthermore, target-absent response time slopes tend to be twice as large as target-absent response slopes. These two characteristics are trademarks of a "serial, selfterminating" search strategy. In Figure 5, an example display is shown from such an experiment, in which the color values have been exchanged for different brightness values.

Unsaturated colors can also be used to study the phenomenon of illusory conjunctions (Treisman \& Schmidt, 1982). For example, if subjects are asked to find a target defined as a "red X," they are more likely to make false reports of target presence when all items are red Os and green Xs than when they are blue Os and green Xs.

Search for isoluminant colors. In a traditional visual search experiment in which color is used as a feature, hue and luminance tend to be covaried (Treisman \& Gelade, 1980). However, recent psychophysical and physiological evidence suggests that there are parallel streams in

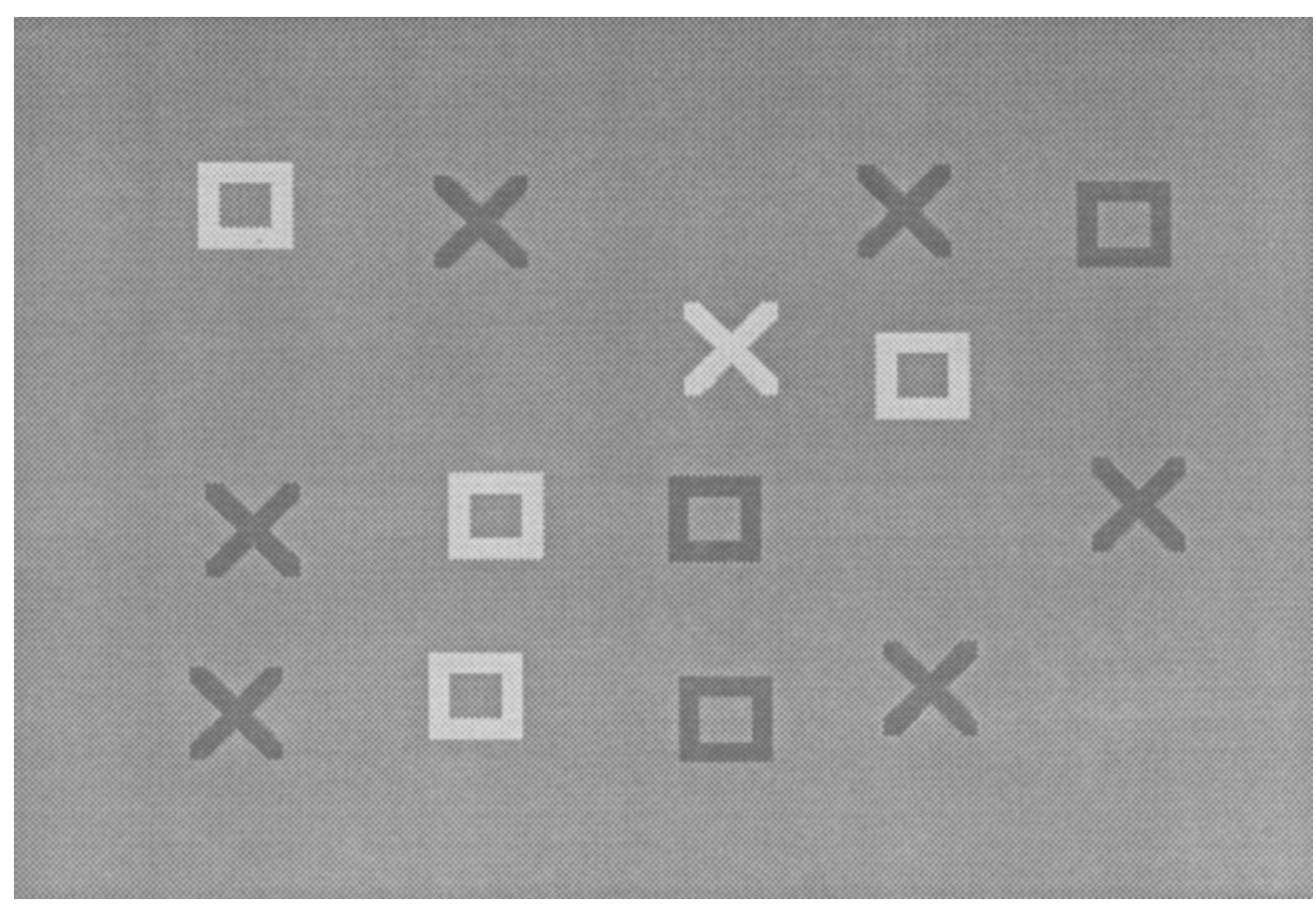

Figure 5. An example display from a search task in which the target is defined by the conjunction of brightness and shape (a light $X$ among light $O$ s and dark $X s$ ). 


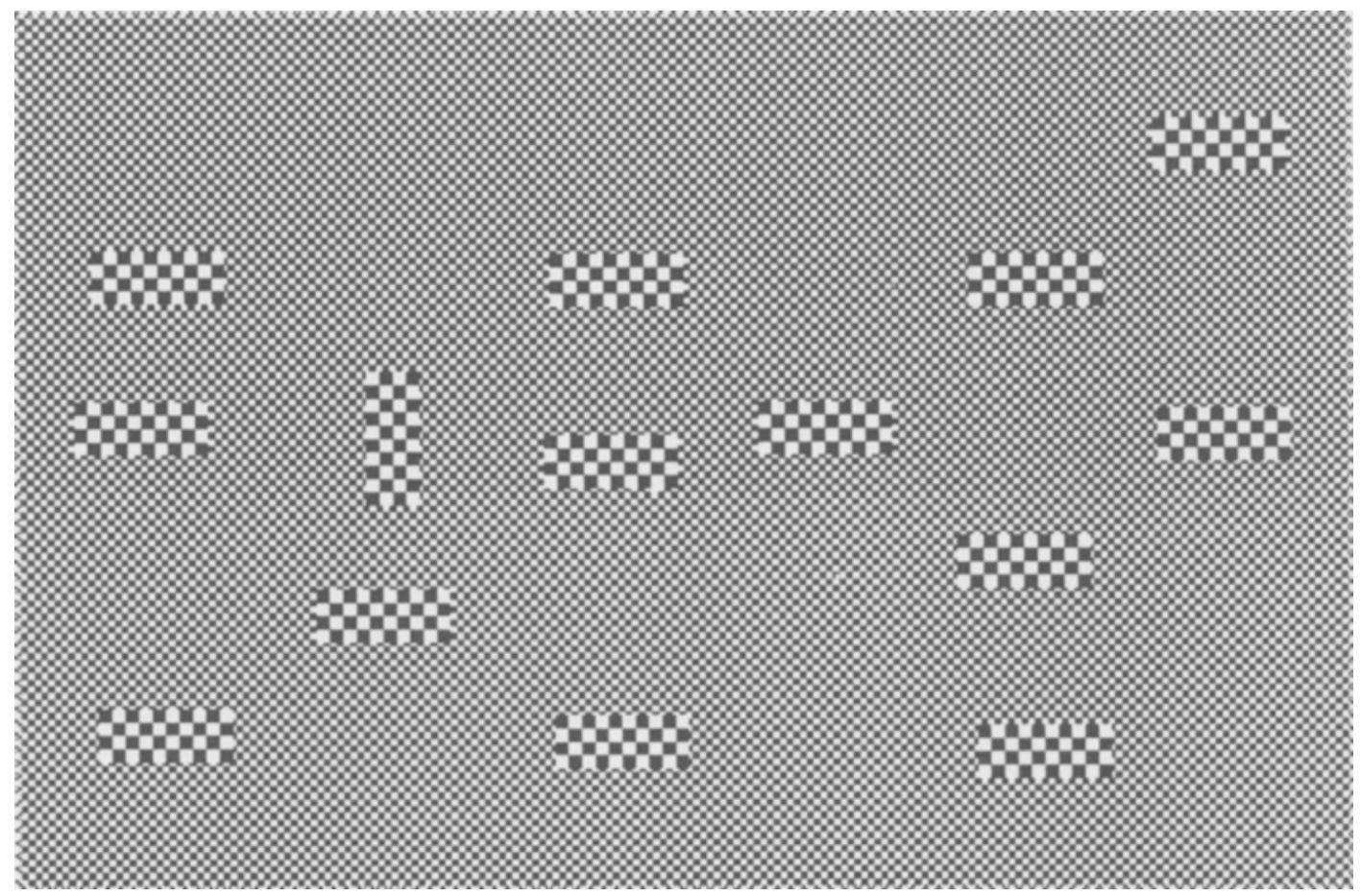

Figure 6. An example display in which the target (a vertical bar) and distractor items (horizontal bar) are defined only by differences in the coarseness of the texture.

the visual cortex for the processing of hue and form (Livingstone \& Hubel, 1987). Thus, it is important to determine whether "pop-out" search is possible for differences in hue alone. Preliminary evidence suggests that it is (Cavanagh, Arguin, \& Treisman, 1990). In Figure 6 , an example display from an experiment involving isoluminance is shown; note that the color values have been exchanged for textures that differ in coarseness.

Search for texture regions defined by color and/or form. A second standard diagnostic of the primitive features of vision is the texture-segregation task. Regions of an image that differ from the background in brightness, hue, or some aspects of form (e.g., orientation of line elements) segregate spontaneously (Beck, 1982; Julesz, 1981). Regions defined by other differences, including some spatial relations between lines, and conjunctions of color and orientation, segregate only with attentional scrutiny (Treisman \& Gelade, 1980). Ramachandran (1988) and Enns and Rensink (1990) have recently shown, however, that some spatial relations in an image do permit "pop-out." For example, spatial relations consistent with deviations from a single lighting direction allow for spontaneous segregation. An example of a display illustrating the sensitivity of early vision to differences in lighting direction is shown in Figure 7.

Search for sinusoidal gratings and Gabor patches. Several models of early form vision are based on spatial filtering (see, e.g., Marr, 1982). This idea can be extended to account for "pop-out" in visual search tasks, or spon- taneous segregation in texture search tasks, by proposing that target and distractor items differ in the spatial filters that they excite (see, e.g., Fogel \& Sagi, 1989; Malik \& Perona, 1989). The stimuli used to test these ideas are often sinusoidally modulated gratings (Shulman $\&$ Wilson, 1987), square-wave gratings (Steinman, 1987), or Gabor patches (Sagi, 1988). An example of a display from a test of orientation sensitivity is shown in Figure 8.

Search for realistically rendered objects. The luminance value of each pixel in an image of objects in a 3-D scene is determined by four properties: lighting, surface reflectance, surface orientation, and viewpoint. Many 3-D drawing and design programs are now available for the Macintosh that enable the drawings of objects with realistic shading and perspective rendering. This permits more finely grained tests of the hypothesis that early vision is sensitive to scene-based properties (Enns \& Rensink, 1990). An example display from a test of texture segregation via 3-D object orientation is shown in Figure 9.

\section{New Paradigms Supported by VSearch Color}

In this section, we give several examples of the variations on the basic visual search task that are now possible with VSearch Color. (The reader should note again that this list is illustrative and not meant to be exhaustive.)

Visual identification experiments. In many visual experiments, the desired response from the subject involves not whether a particular stimulus item is present or absent (detection), but whether one or another stimuli is present 


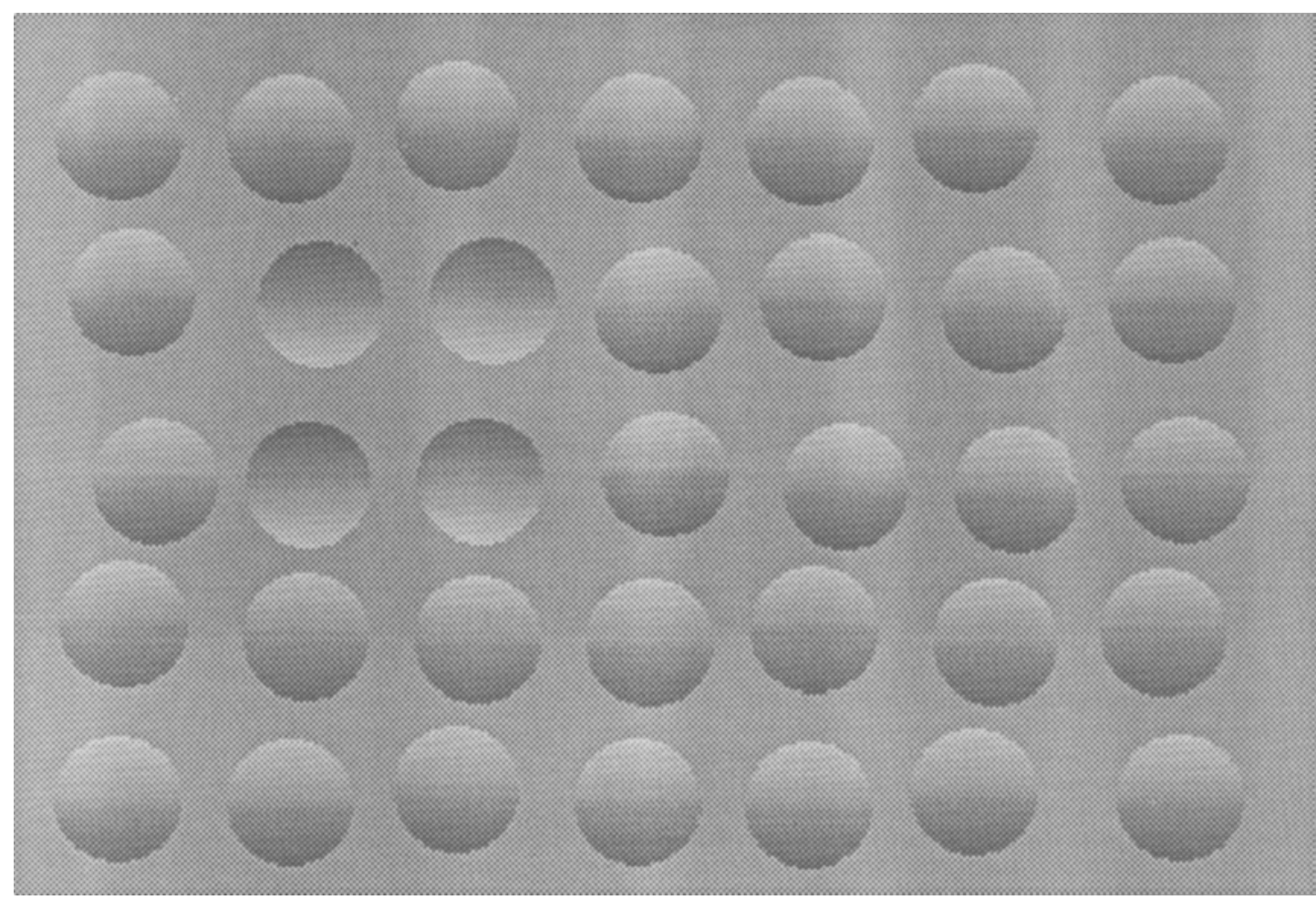

Figure 7. An example display in which a textured region is defined by a $180^{\circ}$ difference in the direction of lighting of the spherical elements.

(identification). VSearch Color permits the implementation of either a two-alternative or a three-alternative forced choice task of this sort. However, in order to maintain the speed and efficiency of the existing program, users are limited to a maximum of three different target items and three different distractor items. This is consistent with our goal of keeping the program an easy-to-use, albeit specialized, tool for visual search experiments.

This new response possibility makes it very easy to design identification experiments in which there are a small number of possible stimuli. Examples include some wordsuperiority experiments (Baron \& Thurstone, 1973) and object-superiority experiments (Enns \& Prinzmetal, 1984; Weisstein \& Harris, 1974). A postdisplay pattern mask (e.g., a haphazard set of lines) is often used in these experiments to set accuracy at a certain level. This is easily done with the Mask|Cue dialogue box.

Number discrimination experiments. Visual enumeration seems to involve two processes. When four or fewer items are present in a display, the apprehension of number is effortless; we simply "see" the number of items. This is called "subitizing." Enumerating larger numbers of items, which is much more laborious, is called "counting" (Trick \& Pylyshyn, 1988). When faced with large numbers of items, many subjects group items into small clusters of two to five. They then move their attention from cluster to cluster, adding the number of each successive cluster into a running total (van Oeffelen \& Vos, 1982).

Number discrimination tasks involving either two or three alternatives can be designed easily with VSearch Color. A region identification task may be specified in which the items to be counted are associated with different regions. The number of target items in each region can be set independently of the number of distractors in the same region and independently of its spatial dimensions.

Adaptation experiments. One of the standard tests used to study the "channels" of early vision is the adaptation test. Adaptation is accomplished by having the observer view an adapting pattern for several seconds or minutes. Subjects can then be tested in a discrimination test with and without prior adaptation. If the adaptation period has an influence on discrimination, the adapting and test stimuli are assumed to be processed with the same "channels."

VSearch Color permits an adapting stimulus to be placed in each of the masking fields that appear between trials in the visual search task. This adapting field may contain a single color value or a tiled pattern of mask items. Duration of the adapting field on each trial is adjustable by the user and can be as long as $15 \mathrm{sec}$. In addition, it is 


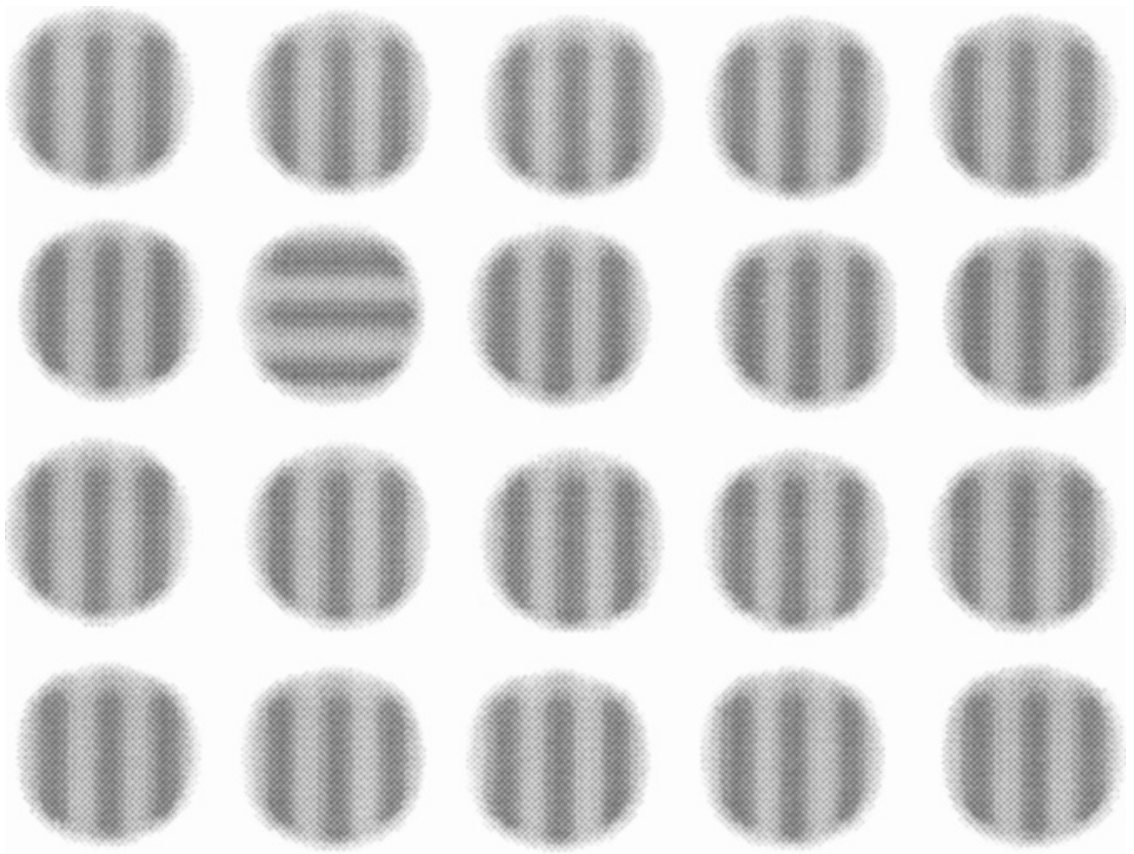

Figure 8. An example display in which the target is a nitered patch defined by orientation.

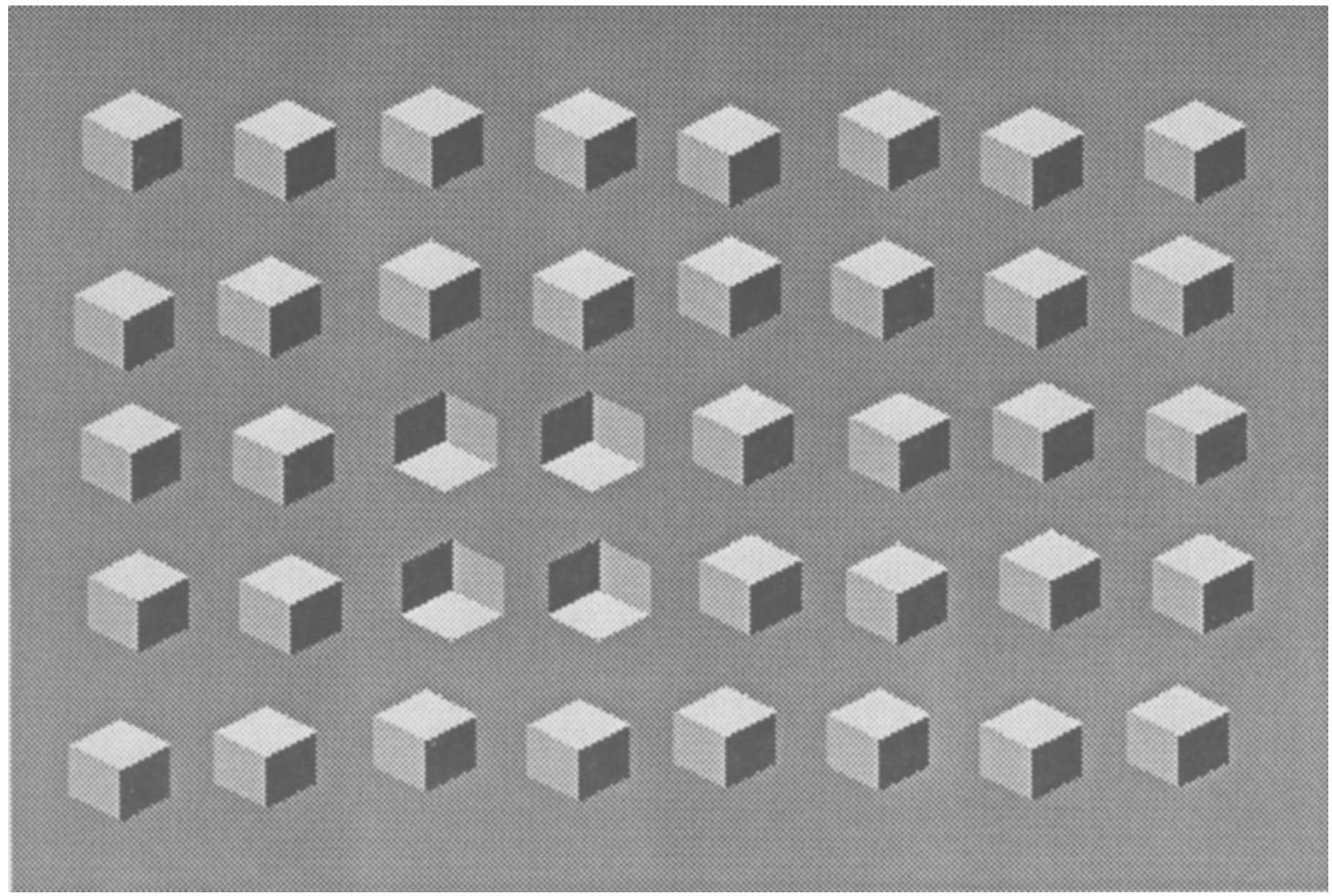

Figure 9. An example display in which the target region is defined by a difference in 3-D orientation. 
also possible to show the masking fields for an indefinite period of time by using the Look function.

Speed-accuracy tradeoff experiments. It is often important to study systematically the relation between the speed and accuracy of a response to a stimulus (Wickelgren, 1977). A tone or visual pattern can be set up to occur at up to eight different intervals following the presentation of a visual display. Subjects can be trained to hold or hurry their decision in response to the signal. The response time and accuracy data collected in this way can then be examined for the shape of the function relating accuracy to speed, the threshold speed at which accuracy exceeds chance levels, and the asymptotic accuracy that can be achieved under various conditions.

A second use of the tone is to study search performance with a random warning foreperiod. The role played by temporal certainty/uncertainty in performance can be studied by comparing a variable-length preparation period with a fixed preparation period.

Masking and cuing experiments. Pre- and postdisplay masks can be used to interrupt visible persistence of the stimulus (sometimes called iconic memory) that continues for some time after the stimulus has been physically terminated. This procedure makes it possible to study the relation between processing time and access to various visual features (Sperling, 1960), the recovery of 3-D information (Epstein, Hatfield, \& Muise, 1977), and individual differences in representational quality (Loftus \& Hanna, 1989).

Spatial cuing can be used to study the visual system's response to the presence of sudden change in the visual field. For example, if there is a sudden change in brightness, the center of gaze is automatically drawn to this location. However, even before the eye has an opportunity to move physically (this takes at least $200 \mathrm{msec}$ on the average), processing of visual information at the location is enhanced. This covert orienting of attention can be studied by examining the way in which detection and identification tasks change as a function of cue validity, cue type, and the temporal characteristics of the cue-target interval (Posner, 1980).

\section{Availability}

A VSearch Color demo disk that illustrates all of the features we have discussed (with the exception that it does not permit the printing or storing of data) is available from the first author at no cost. The complete VSearch Color package (Version 3.1, December 1990) is available for $\$ 300$ ( $\$ 350$ Canadian) to first-time customers and for $\$ 100$ ( $\$ 100$ Canadian) to customers of earlier versions of VSearch. The package includes a manual of over 80 pages with detailed descriptions of how to implement these and other experiments.

\section{REFERENCES}

BARON, J., \& THURSTONe, I. (1973). An analysis of the word-superiority effect. Cognitive Psychology, 4, 207-228.

BeCK, J. (1982). Textural segmentation. In J. Beck (Ed.), Organization and representation in perception (pp. 285-317). Hillsdale, NJ: Erlbaum.

Cavanagh, P., Arguin, M., \& Treisman, A. (1990). Effect of surface medium on visual search for orientation and size features. Journal of Experimental Psychology: Human Perception \& Performance, 16, 479-491.

ENNs, J. T., OChs, E. P., \& Rensink, R. A. (1990). VSearch: Macintosh software for experiments in visual search. Behavior Research Methods, Instruments, \& Computers, 22, 118-122.

EnNs, J. T., \& Prinzmetal, W. (1984). The role of redundancy in the object-line effect. Perception \& Psychophysics, 35, 22-32.

ENNS, J. T., \& RENSINK, R. A. (1990). Influence of scene-based properties on visual search. Science, 247, 721-723.

Epstein, W., Hatfield, G., \& Muise, G. (1977). Perceived shape at a slant as a function of processing time and processing load. Journal of Experimental Psychology: Human Perception \& Performance, 3, 473-483.

FoGEL, I., \& SAGI, D. (1989). Gabor filters as texture discriminator. Biological Cybernetics, 61, 103-113.

JuLEsz, B. (1981). Textons, the elements of texture perception, and their interactions. Nature, 290, 91-97.

Livingstone, M. S., \& Hubel, D. (1987). Psychophysical evidence for separate channels for the perception of form, color, movement, and depth. Joumal of Neuroscience, 7, 3416-3468.

LofTus, G. R., \& HANNA, A. M. (1989). The phenomenology of spatial integration: Data and models. Cognitive Psychology, 21, 363-397.

Malik, J., \& Perona, P. (1989). A computational model of texture perception. In Proceedings of IEEE Conference on Computer Vision and Pattern Recognition (pp. 326-332). San Diego.

MARR, D. (1982). Vision. San Francisco: W. H. Freeman.

PoSNER, M. I. (1980). Orienting of attention. Quarterly Journal of Experimental Psychology, 32, 3-25.

Ramachandran, V. S. (1988). Perceiving shape from shading. Scientific American, 259, 76-83.

Rensink, R. A. (1990). Toolbox-based routines for Macintosh timing and display. Behavior Research Methods, Instruments, \& Computers, 22, $105-117$.

SAGI, D. (1988). The combination of spatial frequency and orientation is effortlessly perceived. Perception \& Psychophysics, 43, 601-603.

Shulman, G. L., \& Wilson, J. (1987). Spatial frequency and selective attention to spatial location. Perception, 16, 103-111.

SPERLING, G. (1960). The information available in brief visual presentations. Psychological Monographs, 74(11, Whole No. 498).

Steinman, S. B. (1987). Serial and parallel search in pattern vision? Perception, 16, 389-398.

Treisman, A., \& Gelade, G. (1980). A feature integration theory of attention. Cognitive Psychology, 12, 97-136.

Treisman, A., \& SCHMidT, H. (1982). Ilusory conjunctions in the perception of objects. Cognitive Psychology, 14, 107-141.

TRICK, L., \& PYLYSHYN, Z. W. (1988). When subitizing fails: The importance of preattentive item indexing for subitizing (Cognitive Science Memorandum No. 35). London, Ontario, Canada: University of Western Ontario, Department of Psychology.

van OefFelen, M. P., \& Vos, P. G. (1982). Configurational effects on the enumeration of dots: Counting by groups. Memory \& Cognition, 10, 396-404.

WeISSTEIN, N., HarRIS, C. S. (1974). Visual detection of line segments: An object-superiority effect. Science, 186, 752-755.

WICKELGREN, W. A. (1977). Speed-accuracy tradeoff and information processing dynamics. Acta Psychologica, 41, 67-85. 